\title{
Prospective comparison of differences
}

\section{in bone mineral density adjacent to two biomechanically different types of cementless femoral stems}

Accepted: 26 November 2003 / Published online: 17 January 2004 (C) Springer-Verlag 2004

\begin{abstract}
We compared the differences in bone mineral density (BMD) adjacent to two biomechanically different cementless femoral stems (Spotorno and Evolution-K). Measurements were performed within the first week after surgery and $3,6,12$, and 24 months postoperatively in a prospective study of 31 patients with 37 total hip arthroplasties. A modified Harris hip score and a visual analogue score for patient satisfaction was used to evaluate the clinical outcome. For both stems, dual Xray absorptiometry measurements revealed a decrease in BMD in Gruen zone 7. Patients with a Spotorno stem also had a significant decrease in BMD in zones 1 and 2. In patients with an Evolution-K stem, there was a positive correlation between the clinical outcome and BMD in Gruen zones 1 and 4. Our data suggest that periprosthetic bone loss is equal to if not less pronounced adjacent to the Evolution-K stem as compared to the Spotorno stem.
\end{abstract}

Résumé Nous avons comparé les différences dans les densités minérales osseuses (DMO) au niveau de deux tiges fémorales sans ciment de conception biomécanique différente (Spotorno et Evolution-K). Les mesures ont été exécutées dans la première semaine après chirurgie et à $3,6,12$, et 24 mois postopératoires dans une étude prospective de 31 malades avec 37 arthroplasties totales de la hanche. Un score modifié de Harris et un score analogue visuel pour la satisfaction des patients ont été utilisés pour évaluer le résultat clinique. Pour les deux tiges l'examen d'absorptiomètre biphotonique a révélé

\section{B. Zerahn · I.-L. Kanstrup}

Department of Clinical Physiology and Nuclear Medicine,

Herlev Hospital, University of Copenhagen,

Copenhagen, Denmark

\section{G. S. Lausten}

Department of Orthopaedic Surgery, Herlev Hospital, University of Copenhagen, Copenhagen, Denmark

\section{B. Zerahn (๘)}

Gl. Jernbanevej 30, 2.th., 2500 Valby, Denmark

e-mail: bozer@dadlnet.dk

Tel.: +45-3-2595959 une baisse de DMO dans les zones 7 de Gruen. Les malades avec une tige Spotorno avaient aussi une baisse importante de DMO dans les zones 1 et 2 . Chez les malades avec une tige Evolution- $K$ il $y$ avait une corrélation positive entre le résultat clinique et la DMO dans les zones 1 et 4 de Gruen. Nos données suggèrent que la perte minérales osseuses est équivalente au voisinage des deux tiges prothétiques.

\section{Introduction}

Remodelling of bone adjacent to the proximal part of both cemented and cementless stems is considered to be an important factor in the long-term survival of a total hip arthroplasty (THA). In cementless prostheses, the preservation of bone in the proximal femur is particularly important in case of revision. Periprosthetic bone mineral density (BMD, grams per centimeter squared) after THA can be determined with high precision using dual energy $\mathrm{X}$-ray absorptiometry $[2,7]$.

We have previously examined changes in bone mass after insertion of an off-the-shelf, stem-type hip prosthesis (Spotorno CLS, Protek AG, Berne) or a custom-made femoral implant (Evolution-K, Fehling Werke, Tübingen, Germany) in a cross-sectional study [15]. Now we present a prospective study with a 2-year follow-up including a modified Harris hip score.

\section{Materials and methods}

Subjects

Thirty-seven cementless THAs of either the Evolution-K (11 hips in 10 patients) or Spotorno type (26 hips in 21 patients) were included. Informed consent was obtained from all patients, and the investigation was approved by the ethical committee of Copenhagen County, Denmark (KA 96148).

Measurements were performed within a week after surgery and $3,6,12$, and 24 months after surgery. Age, height, weight, and gender were recorded. BMD values from the femur of the operated leg and both proximal tibiae were obtained in all patients. 
Ten hips in the Evolution-K group and 22 in the Spotorno group were replaced because of osteoarthritis. In the Spotorno group, THA was performed in four cases because of avascular necrosis of the femoral head, and one patient had an Evolution-K prosthesis because of hip dysplasia.

Five women and five men had Evolution-K stems. These patients were offered custom-made prosthesis, because the shape or size of the proximal femoral shaft was considered unsuitable for a standard cementless prosthesis. Eleven women and ten men had a Spotorno stem. Three patients in the Evolution-K group and five (seven hips) in the Spotorno group did not perform all scans. All patients were scanned at 24 months postoperatively, except one patient with a Spotorno stem who had emigrated. There were no stem revisions, and no patients died during the follow-up period. However, one patient had the cup replaced because of recurrent dislocations.

All patients except the emigrated patient were scored with a modified Harris hip score, excluding data on range of motion thus leaving a maximum score of 91 . They were also asked to mark their satisfaction on a visual analogue scale (VAS) ranging from zero to 100.

\section{Measurements}

When performing a Gruen analysis, each of the six zones adjacent to the implant were assigned the height of one third of the implant length [12]. For Gruen zone 7 however, the upper limit of the region was set at $3 \mathrm{~mm}$ below the cutting edge of the femoral neck. The BMD value for each Gruen zone is expressed in grams per centimeter squared, reflecting a two-dimensional image of the bone as seen in the anteroposterior plane.

Scanning was performed with the patient in the supine position. The leg was secured in a neutral position by standardized support for foot and thigh. The radiation dose for each hip scan was $0.05 \mathrm{mSv}$. For scanning of the proximal tibia, the scanned leg was supported in a $10^{\circ}$ inwardly rotated position. BMD values were obtained from a $1 \mathrm{~cm}^{2}$ region of interest (ROI) placed below the medial and lateral subchondral plates of the proximal tibia.

A system quality assurance program was performed on a daily and weekly basis according to the manufacturer's recommendations. There were no significant drifts or jumps during the study period. The standard deviation for phantom measurements was $0.08 \mathrm{~g} / \mathrm{cm}^{2}$, or $0.60 \%$. The X-ray tube was replaced once during the study.

Repeated measurements on a cadaver femur with an inserted prosthetic femoral stem were performed to assess variations in reproducibility with rotation. The cadaver femur was placed in a water bath and scanned 17 times in various rotations from $15^{\circ}$ outward rotation to $15^{\circ}$ inward rotation with increments of $5^{\circ}$. Eight patients were scanned twice to assess reproducibility in vivo.

\section{Stem types}

The custom-made femoral stem (Evolution-K) is individually shaped based on a CT scan of the patient's proximal femur. After reaming with an individually adapted reamer of the same size and shape as the prosthesis itself, a perfect press fit of the prosthesis into the femoral canal was obtained in all cases.

The standard femoral stem (Spotorno CLS), which has a double-wedge shape, was inserted by conventional cementless technique. The approximate size of the prosthesis was estimated preoperatively by templates on radiograms of the proximal femur. The femoral stem was fitted with a minimum of $2 \mathrm{~mm}$ impacted cancellous bone surrounding the implant.

In all cases, a cementless standard press-fit acetabular cup with a porous coating (Harris-Galante, Zimmer, Warsaw, IN, USA) was used. Both femoral stems were made of a Ti-Al alloy and had similar sandblasted surface coatings. There was no hydroxyapatite coating.

\section{Statistical analysis}

The Wilcoxon matched pairs test was used for comparing differences in BMD between two scans. A Mann-Whitney $U$ test was used when comparing data between the two stem types. The Friedman test for two-way analysis of variance was used to detect changes in BMD with time within groups. Correlation between Harris hip score, VAS-score, and BMD was calculated by use of Kendall's thau_b for nonparametric correlation. The percentage coefficient of variation (CV\%) for two measurements performed on the same day was assessed according to the following expression:

$\frac{100 \sqrt{\left(\sum d^{2} / 2 n\right)}}{\left(m_{1}+m_{2}\right) / 2}$

where $d$ is the difference between two measurements, $n$ is the number of measurement pairs, and $m_{1}$ and $m_{2}$ are the first and second measurement results respectively.

The CV\% for repeated measurements on the cadaver femur was assessed according to the following expression:

$100 \times \mathrm{SD}_{\mathrm{n}}$

$\left(\sum m_{n}\right) / \mathrm{n}$

where $\mathrm{SD}_{\mathrm{n}}$ is the standard deviation of $n$ repeated measurements. A $p$ value of less than 0.05 was accepted as significant. All analyses were accomplished using the SPSS statistical software version 9.0 (SPSS Inc, Chicago, IL, USA).

\section{Results}

The in vivo reproducibility given in $\mathrm{CV} \%$ for Gruen zones 1-7 was $2.8,2.1,1.7,2.9,1.7,2.3$, and 3.3 respectively. In Gruen zone 7, the mean BMD changed by $2.1 \mathrm{SD}$ for each $5^{\circ}$ of rotation. If rotation was kept within $\pm 5^{\circ}$, the mean BMD value for all other Gruen zones varied less than \pm 2 SD for each increment of rotation.

There were no statistically significant differences between the two groups regarding age, modified Harris hip score, VAS score, height, and body weight (Table 1).

BMD values for each Gruen zone and proximal tibia are listed in Table 2. There were no significant changes in BMD adjacent to the Evolution-K stem except for Gruen zone 7 at 2 years after surgery, although there was a minor decline in BMD of the medial part of the proximal tibia. Adjacent to the Spotorno stem, there was a significant decrease in BMD values in Gruen zones 1,2, and 7 at 2 years after surgery. There was also a trend towards a decrease in BMD in zone 6 adjacent to the Spotorno stem 2 years after surgery.

For patients with Evolution-K stems, there was a positive correlation between both modified Harris hip score and VAS score on one side and BMD of the proximal lateral tibia and in Gruen zones 1 and 4 on the other (Table 3). There were no similar correlations found for patients with Spotorno stems.

Table 1 Median values (range) for patients' preoperative age, height, body weight, and Harris hip score and visual analog scale (VAS) score at 24 months after surgery

\begin{tabular}{lcc}
\hline & Evolution-K & Spotorno \\
\hline Age (years) & $51(32-64)$ & $53(38-65)$ \\
Height $(\mathrm{cm})$ & $175(160-192)$ & $173(154-188)$ \\
Body weight $(\mathrm{kg})$ & $82(55-102)$ & $84(60-115)$ \\
Modified Harris hip score & $89(54-91)$ & $87(62-91)$ \\
VAS score & $95(50-100)$ & $90(50-100)$ \\
\hline
\end{tabular}


Table 2 Median values (range) for bone mineral density (BMD) in the operated leg in patients with Evolution-K or Spotorno stems

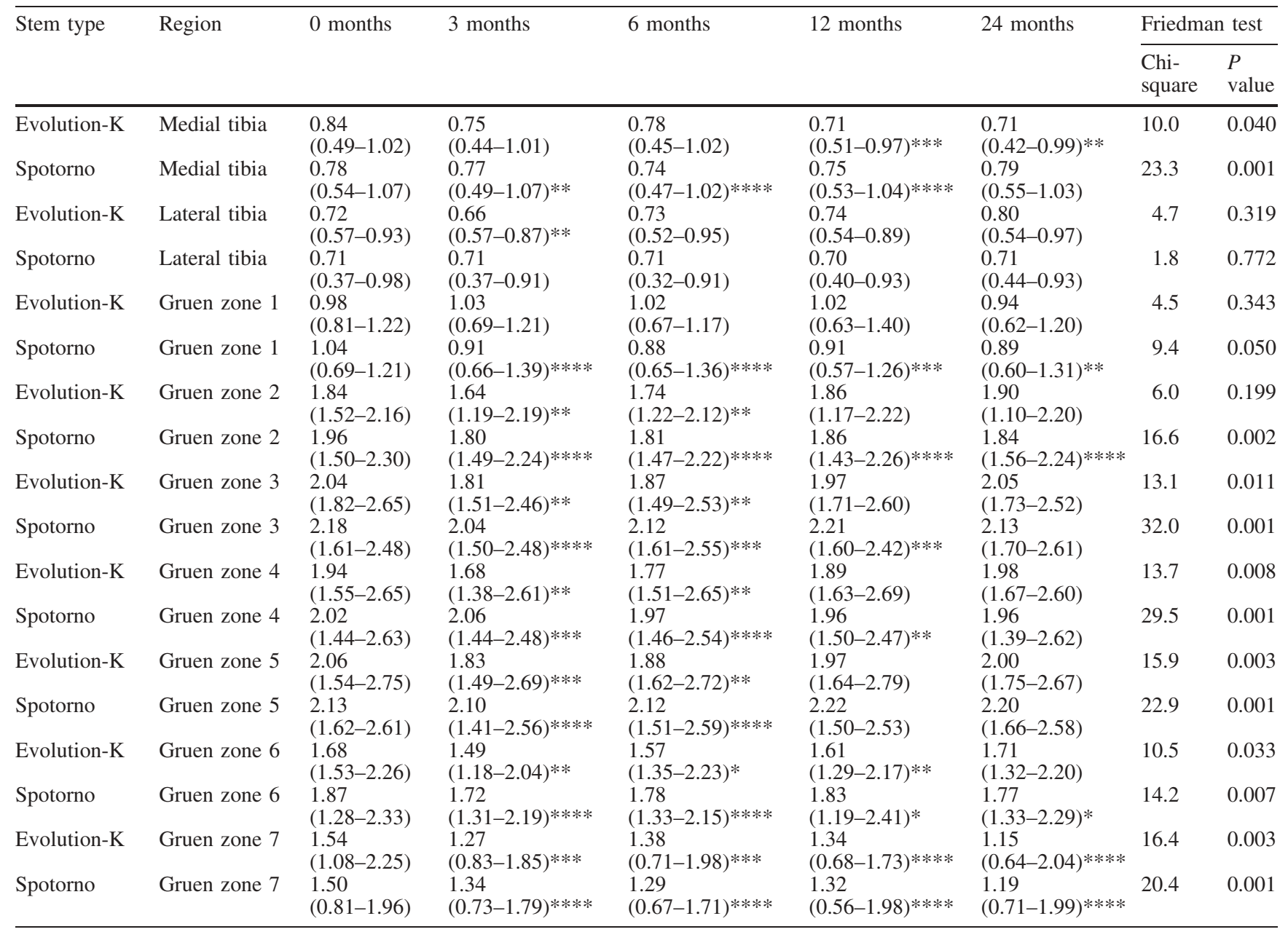

BMD values are given in grams per centimeter squared

$P$ values are given for comparison between immediate postoperative values versus postoperative values at $3,6,12$, and 24 months by use of the Wilcoxon matched pairs test

$* 0.1>p>0.05$

$* * 0.05>p>0.02$

$* * * 0.02>p>0.01$

$* * * * p \leq 0.01$

Chi-square and $p$-values for Friedman's two-way analysis of variance are given for changes in BMD with time after surgery

Table 3 Correlation coefficients and significance levels for the relation between visual analog scale (VAS) score, modified Harris Hip score and bone mineral density (BMD) values 24 months after surgery

\begin{tabular}{|c|c|c|c|c|}
\hline \multirow[t]{2}{*}{ BMD } & \multicolumn{2}{|c|}{ Modified Harris hip score } & \multicolumn{2}{|l|}{ VAS score } \\
\hline & Evolution-K & Spotorno & Evolution-K & Spotorno \\
\hline Gruen zone 1 & $0.636 * * *$ & 0.015 & $0.583 * *$ & -0.028 \\
\hline Gruen zone 2 & 0.278 & -0.026 & 0.342 & -0.076 \\
\hline Gruen zone 3 & 0.199 & 0.058 & 0.141 & -0.062 \\
\hline Gruen zone 4 & $0.517 *$ & 0.120 & $0.623 * *$ & -0.003 \\
\hline Gruen zone 5 & 0.239 & -0.055 & 0.302 & -0.003 \\
\hline Gruen zone 6 & -0.080 & 0.164 & -0.221 & 0.058 \\
\hline Gruen zone 7 & 0.080 & 0.128 & 0.221 & 0.093 \\
\hline Lateral tibia & $0.596 * *$ & 0.070 & $0.623 * *$ & -0.041 \\
\hline Medial tibia & 0.239 & -0.128 & 0.141 & 0.031 \\
\hline
\end{tabular}




\section{Discussion}

Dual-energy X-ray absorptiometry is a reproducible method for quantitative analysis of BMD adjacent to metallic implants. Our results confirm that the accuracy of the method is as good as found in several other studies [2, $6,7,11,13]$. However, careful positioning is mandatory in particular for measurements in Gruen zone 7 [10].

Our two groups were comparable regarding gender, age, height, and body weight. Consequently, these factors were not expected to have a significant impact on the difference between the two groups regarding changes in BMD. Two years after surgery, neither the modified Harris hip score nor the VAS score differed between the two stem types. For obvious ethical reasons, the patients could not be randomized. Comparing the two groups is still relevant, since the only other option for the patients with the Evolution-K stem would be a cemented femoral stem.

As compared to our previous results from a crosssectional study [15], we have now confirmed a BMD decrease in Gruen zones 1, 2, and 7 and a decrease trend in Gruen zone 6 for the Spotorno prosthesis. For the Evolution-K prosthesis, we only found a significant decrease in BMD in Gruen zone 7, which was not significantly present in the first study. Nor have we been able to confirm an increase in BMD in Gruen zone 5. Thus, apparently the Evolution-K stem is superior in preserving bone mass in the proximal lateral aspect of the femur. In the Evolution-K group there was a reduction of BMD beneath the medial subchondral plate in the proximal tibia and a corresponding tendency towards an increase in BMD beneath the lateral subchondral plate. This indicates a change in the loading of the operated leg with a relative lateralization of the proximal femur giving increased weight bearing on the lateral part of the knee joint. Apparently, there may be an association between this lateralization and the clinical results, since there is a positive correlation between VAS and Harris hip scores and BMD levels of the lateral tibia and femur (Gruen zones 1 and 4). In the Spotorno group there was no significant decrease in BMD of the proximal tibia. Thus, the bone loss adjacent to the Spotorno prosthesis cannot be accounted for by a decreased loading of the leg [1].

During the first postoperative year, there were marked declines in BMD in most Gruen zones adjacent to both stems. At 2 years after surgery, the most distal of the Gruen zones had recovered their preoperative bone mass in contrast to the proximal regions. In particular, Gruen zone 7 is prone to a continuous loss of bone mass. Engh et al. [4] have shown that the remodeling of the proximal femur after insertion of cementless prostheses mainly takes place during the first 2 postoperative years. The changes in bone mass at 2 years follow-up are therefore likely to be permanent. Dickob [3] found an average decrease in bone mass of $26 \%$ adjacent to the Spotorno CLS stem, which is comparable only to the decline in bone mass in Gruen zone 7 (21\%) in our study. Results from a study by Gibbons [5] are similar to ours on reduction of bone mass adjacent to the CLS stem, showing a reduction of bone mass in region 7 of $20.5 \%$. In a cross-sectional study, Martini [9] found a significant loss of BMD ranging from 5 to $21 \%$ in all seven Gruen zones in 23 patients with Evolution-K stems. The mean follow-up period was 21.2 (3-83) months. They interpreted their data in support of a hypothesis that the decline in bone mass during the first postoperative year will affect the outcome of the study. They found a bone loss in Gruen zone 7 of $21 \%$, or $0.3 \mathrm{~g} / \mathrm{cm}^{2}$, which is in accordance with our findings $\left(25 \%\right.$, or $\left.0.39 \mathrm{~g} / \mathrm{cm}^{2}\right)$ at 2 years' follow-up. Nevertheless, bone loss adjacent to the Evolution-K and CLS stem appears to be comparatively low in most studies.

Since declines in bone mass adjacent to femoral implants are associated with both high bone turnover [8] and loosening of the stem [14], the modest decrease of bone mass adjacent to the Evolution-K stem is very likely to be an advantage, or at least comparable, to the Spotorno stem.

In conclusion, we have confirmed that the custommade femoral stem preserves adjacent bone in the proximal lateral regions of the femur as well as, if not better than, the off-the-shelf-type cementless femoral stem. There is a correlation between the preservation of bone mass in the proximal lateral aspect of the femur and the clinical outcome of the THA. This indicates that the Evolution- $\mathrm{K}$ design is a sufficient alternative in younger patients who otherwise would need to have a cemented hip prosthesis. However, long-term clinical results are still needed to support such a conclusion.

\section{References}

1. Bryan JM, Sumner DR, Hurwitz DE, Tompkins GS, Andriacchi TP, Galante JO (1996) Altered load history affects periprosthetic bone loss following cementless total hip arthroplasty. J Orthop Res 14:762-768

2. Cohen B, Rushton N (1995) Accuracy of DEXA measurement of bone mineral density after total hip arthroplasty. J Bone Joint Surg [Br] 77:479-483

3. Dickob M, Schnittko A, Pfandl S, Wetzel R, Puhl W (1993) Die DXA-Methode zur Quantifizierung von Knochendichteänderungen des Femurs nach zementfreier Hüftenprothetik. Osteologie 2:206-212

4. Engh CA, Hooten JP Jr, Zettl-Schaffer KF, Ghaffarpour M, McGovern TF, Macalino GE, Zicat BA (1994) Porous-coated total hip replacement. Clin Orthop 298:89-96

5. Gibbons CE, Davies AJ, Amis AA, Olearnik H, Parker BC, Scott JE (2001) Periprosthetic bone mineral density changes with femoral components of differing design philosophy. Int Orthop 25:89-92

6. Kiratli BJ, Heiner JP, McBeath AA, Wilson MA (1992) Determination of bone mineral density by dual X-ray absorptiometry in patients with uncemented total hip arthroplasty. J Orthop Res 10:836-844

7. Kroger H, Miettinen H, Arnala I, Koski E, Rushton N, Suomalainen O (1996) Evaluation of periprosthetic bone using dual-energy X-ray absorptiometry: precision of the method and effect of operation on bone mineral density. J Bone Miner Res 11:1526-1530

8. Kroger H, Vanninen E, Overmyer M, Miettinen H, Rushton N, Suomalainen O (1997) Periprosthetic bone loss and regional 
bone turnover in uncemented total hip arthroplasty: a prospective study using high resolution single photon emission tomography and dual-energy X-ray absorptiometry. J Bone Miner Res 12:487-492

9. Martini F, Sell S, Kremling E, Küsswetter W (1996) Determination of periprosthetic bone density with the DEXA method after implantation of custom-made uncemented femoral stems. Int Orthop 20:218-221

10. Mortimer ES, Rosenthall L, Paterson I, Bobyn JD (1996) Effect of rotation on periprosthetic bone mineral measurements in a hip phantom. Clin Orthop 324:269-274

11. Sabo D, Reiter A, Simank HG, Thomsen M, Lukoschek M, Ewerbeck V (1998) Periprosthetic mineralization around cementless total hip endoprosthesis: longitudinal study and cross-sectional study on titanium threaded acetabular cup and cementless Spotorno stem with DEXA. Calcif Tissue Int 62:177-182
12. Sarmiento A, Gruen TA (1985) Radiographic analysis of a lowmodulus titanium-alloy femoral total hip component. Two-tosix-year follow-up. J Bone Joint Surg [Am] 67:48-56

13. Trevisan C, Bigoni M, Cherubini R, Steiger P, Randelli G, Ortolani S (1993) Dual X-ray absorptiometry for the evaluation of bone density from the proximal femur after total hip arthroplasty: analysis protocols and reproducibility. Calcif Tissue Int 53:158-161

14. Venesmaa P, Kroger H, Miettinen H, Jurvelin J, Suomalainen O, Alhava E (2000) Bone loss around failed femoral implant measured by dual-energy X-ray absorptiometry. J Orthop Sci $5: 380-384$

15. Zerahn B, Storgaard M, Johansen T, Olsen C, Lausten G, Kanstrup IL (1998) Changes in bone mineral density adjacent to two biomechanically different types of cementless femoral stems in total hip arthroplasty. Int Orthop 22:225-229 